\title{
BENDING OF LAMINATED PLATES WITH MIXED BOUNDARY CONDITIONS BASED ON HIGHER-ORDER SHEAR DEFORMATION THEORY
}

\author{
Mojgan Yaghoubshahi And Hossein Rajaie
}

\begin{abstract}
The bending of laminated plates is considered using higher-order transverse shear deformation theory. The principle of virtual work is used to derive a new set of seven governing equations and corresponding boundary conditions. These equations, combined with eighteen relationships between the resultant stress and displacement components, compose a system of first-order partial differential equations that is solved by the generalized differential quadrature method. Numerical results for laminated plates with a variety of mixed boundary conditions are calculated using the proposed method, and good agreement is found with the corresponding solutions obtained using ANSYS.
\end{abstract}

\section{Introduction}

Fiber-reinforced laminated composite materials are widely used in a variety of engineering fields, such as aerospace, civil, marine, mechanical, nuclear, and petrochemical engineering. Such materials are popular for industrial applications due to high strength-to-weight ratios, long fatigue life, good stealth characteristics, and enhanced corrosion resistance. A number of theories describing laminated composite plates exist in the literature.

Classical plate theory is based on the Kirchhoff kinematic hypothesis that straight lines normal to the undeformed midsurface remain straight and normal to the middle surface after deformation and undergo no thickness stretching. Neglecting transverse shear effects, this theory produces unacceptable approximations in the analysis of even thin laminated plates and shells. Surveys of various classic shell theories can be found in [Naghdi 1956; Bert and Francis 1974; Bert and Chen 1978].

The development of plate theories with transverse shear effects has improved the accuracy of results considerably. The refined theories are of different orders, based on discretization of the transverse shear effects and the number of terms included in the assumed displacement field. Reissner [1945] was the first to develop a plate theory that included transverse shear deformation for static analysis. Mindlin [1951] then expanded Reissner's theory for dynamic analysis. Both approaches rely on first-order shear deformation theory (FSDT). Reissner's theory is stress-based, whereas Mindlin's is displacement-based. These theories do not satisfy the condition of zero transverse shear stress at the top and bottom surfaces of the plate, and consider a uniform transverse shear stress distribution across the thickness of the plate. Therefore they require the use of a shear correction factor to increase the precision of the results.

Later a set of theories, generally known as higher-order shear deformation theories (HSDT), has been developed by a number of researchers. Basset [1890] appears to have been the first researcher to suggest that displacement fields can be expanded in a power series of the thickness coordinate. The higher-order

Keywords: laminated plates, higher-order transverse shear deformation theory, mixed boundary conditions, generalized differential quadrature method. 
theory presented by Reddy and Liu [1985] is based on a displacement field in which the displacements in the surface of the shell are expanded as a quadratic function of the thickness coordinate. Actually, the higher-order theories require additional computation with respect to the first-order theories.

Employing such theories results in systems of highly coupled partial differential equations. Several methods exist for obtaining solutions for such systems. Among those numerical studies, the differential quadrature (DQ) method, introduced by Bellman et al. [1972], is an efficient method for obtaining accurate numerical results using a few grid points. DQ approximates the spatial derivative of a function with respect to a given coordinate at a discrete point as the weighted linear sum of all functional values in the domain of that coordinate direction. Two methods were proposed by those authors for obtaining the weighting coefficients of the first-order derivative: the first method solves a system of algebraic equations to determine the weighting coefficients, and the second utilizes a simple algebraic formulation, provided that the coordinates of grid points are chosen to be the roots of the shifted Legendre polynomial. The first method is simpler to apply, but the second is more efficient. For the first method, in which the coordinates of grid points are arbitrarily chosen, Quan and Chang [1989] used Lagrange interpolation polynomials as test functions to develop explicit formulations for determining the weighting coefficients for the first- and second-order derivative discretization.

A generalized differential quadrature (GDQ) method was introduced in [Shu and Richards 1990; Shu 1991]. It generalized all current methods via analysis of a higher-order polynomial approximation and analysis of a linear vector space. In GDQ, the weighting coefficients of the first derivatives are determined by a simple algebraic formulation, and there is no restriction on the coordinates of the grid points. The weighting coefficients of the second and higher-order derivatives are determined by a recurrence relationship.

Bert et al. [1988] were the first to apply the DQ method to structural mechanics problems. Subsequently, a number of researchers utilized this method to solve a variety of structural problems relevant to thin plate theory [Striz et al. 1988; Bert et al. 1989; Sherbourne and Pandey 1991]. A bending analysis of thin/thick laminated plates with various boundary conditions based on first-order shear deformation theory was described in [Aghdam et al. 2006]. The DQ method was used in [Li and Cheng 2005; Tornabene and Viola 2008] to solve for the vibration of plates and shells. In [Malekzadeh and Setoodeh 2007], large deformation of laminated plates on a nonlinear elastic foundation was analyzed by the DQ method.

The present study deals with the bending analysis of thin/thick laminated plates with various boundary conditions based on the higher-order shear deformation theory, in which the displacement field presented by Reddy and Liu [1985] is considered. Introducing two new unknown functions $w_{i}(i=1,2)$ for computational purposes, a new set of seven governing equations and corresponding boundary conditions for each edge are derived. Applying the $w_{i}$ allows the governing equations and boundary conditions to be easily obtained from the virtual work formulation. These equations, together with eighteen relationships between the resultant stress and displacement components, form a system of 25 first-order partial differential equations. Solving a set of 25 equations simultaneously enables one to apply boundary conditions in the HSDT more accurately and conveniently. In the HSDT of Reddy and Liu, the governing equations are second-order partial differential equations, and the boundary conditions are first-order partial differential relationships. By applying this technique, the governing equations convert first-order and boundary conditions into linear algebraic relationships. Application of linear algebraic relationships as boundary 
conditions is much easier, and the number of grid points required for convergence in the GDQ method is reduced.

In this paper, plates with free edge and mixed boundary conditions are considered. The results are compared with those obtained ANSYS version 5.4. Numerical results are presented to understand the complex deformation behavior of symmetric and antisymmetric cross ply plates.

\section{Formulation}

A rectangular plate with different boundaries in the $\alpha_{1}$ and $\alpha_{2}$ directions is considered. The principle of virtual work for the equilibrium of a body with surface $S$ and volume $V$ requires satisfaction of

$$
\int_{V} \sum_{i=1}^{3} \sum_{j=1}^{3} \sigma_{i j} \delta \varepsilon_{i j} d V-\int_{S} \delta W_{\mathrm{ext}} d s=0,
$$

where $\sigma_{i j}$ denotes the stress components, $\delta \varepsilon_{i j}$ the variation of virtual strain components caused by virtual displacements, and $\delta W_{e x t}$ the variation in virtual work performed by the external forces. Employing a higher-order shear deformation theory, the displacement components, in terms of functions specifying the deformation of the middle surface of the plate, may be approximated as [Reddy and Liu 1985]

$$
\begin{aligned}
& U_{i}\left(\alpha_{1}, \alpha_{2}, \zeta\right)=u_{i}\left(\alpha_{1}, \alpha_{2}\right)+\zeta \varphi_{i}\left(\alpha_{1}, \alpha_{2}\right)+\zeta^{2} \psi_{i}\left(\alpha_{1}, \alpha_{2}\right)+\zeta^{3} \theta_{i}\left(\alpha_{1}, \alpha_{2}\right), \quad i=1,2 \\
& W\left(\alpha_{1}, \alpha_{2}, \zeta\right)=w\left(\alpha_{1}, \alpha_{2}\right),
\end{aligned}
$$

where, $\zeta$, ranging from $-h / 2$ to $h / 2$, is the variable in the thickness direction.

Convention. Henceforth, unless otherwise stated, we use the subscript $n$ to refer to the normal direction (i.e., $n=3$ ) and $i, j$ are distinct indices ranging over the in-plane directions (i.e., $i=1,2$ and $j=3-i$ ).

One may assume, without loss of generality, that shear stress is absent from the top and bottom surfaces of the plate. Therefore,

$$
\tau_{\text {in }}\left(\alpha_{1}, \alpha_{2}, \pm \frac{h}{2}\right)=0
$$

Hooke's law for a laminated plate composed of orthotropic layers implies that (3) is equivalent to

$$
\gamma_{\text {in }}\left(\alpha_{1}, \alpha_{2}, \pm \frac{h}{2}\right)=0
$$

The strain-displacement relationships in the principal coordinates of a plate are

$$
\varepsilon_{i i}=\frac{\partial U_{i}}{\partial \alpha_{i}}, \quad \varepsilon_{n n}=\frac{\partial W}{\partial \zeta}, \quad \gamma_{i j}=\frac{\partial U_{j}}{\partial \alpha_{i}}+\frac{\partial U_{i}}{\partial \alpha_{j}}, \quad \gamma_{i n}=\frac{\partial W}{\partial \alpha_{i}}+\frac{\partial U_{i}}{\partial \zeta} .
$$

Substitution of (2) into (5) yields

$$
\gamma_{i n}=\frac{\partial w}{\partial \alpha_{i}}+\varphi_{i}+2 \zeta \psi_{i}+3 \zeta^{2} \theta_{i}
$$

Application of (4) to (6) gives the four equations

$$
\left\{\begin{array}{l}
\partial w / \partial \alpha_{i}+\varphi_{i}+h \psi_{i}+\frac{3}{4} h^{2} \theta_{i}=0 \\
\partial w / \partial \alpha_{i}+\varphi_{i}-h \psi_{i}+\frac{3}{4} h^{2} \theta_{i}=0
\end{array} \quad i=1,2 .\right.
$$


Solving the system of algebraic equations (7) yields

$$
\psi_{i}=0, \quad \theta_{i}=-\frac{4}{3 h^{2}}\left(\frac{\partial w}{\partial \alpha_{i}}+\varphi_{i}\right) .
$$

Substituting (8) into (2) gives the displacement components in the thickness direction in terms of displacements of the middle surface of the plate:

$$
U_{i}\left(\alpha_{1}, \alpha_{2}, \zeta\right)=u_{i}+\zeta \varphi_{i}-\zeta^{3} \frac{4}{3 h^{2}}\left(\frac{\partial w}{\partial \alpha_{i}}+\varphi_{i}\right), \quad W\left(\alpha_{1}, \alpha_{2}, \zeta\right)=w\left(\alpha_{1}, \alpha_{2}\right) .
$$

The strain-displacement equations (7), in view of (9), give

$$
\begin{aligned}
\varepsilon_{i i} & =\varepsilon_{i}^{0}+\zeta \kappa_{i}^{0}+\zeta^{3} \kappa_{i}^{2}, & \gamma_{i n} & =\lambda_{i}^{0}+\zeta^{2} \eta_{i}^{1}, \\
\varepsilon_{n n} & =\frac{\partial w}{\partial \zeta}, & \gamma_{i j} & =\gamma_{i}^{0}+\zeta \mu_{i}^{0}+\zeta^{3} \mu_{i}^{2}+\gamma_{j}^{0}+\zeta \mu_{j}^{0}+\zeta^{3} \mu_{j}^{2},
\end{aligned}
$$

where we have set

$$
\begin{aligned}
\varepsilon_{i}^{0}=\frac{\partial u_{i}}{\partial \alpha_{i}}, & \kappa_{i}^{0}=\frac{\partial \varphi_{i}}{\partial \alpha_{i}}, & \kappa_{i}^{2}=-\frac{4}{3 h^{2}} \frac{\partial}{\partial \alpha_{i}}\left(w_{i}+\varphi_{i}\right), & \lambda_{i}^{0}=\frac{\partial w}{\partial \alpha_{i}}+\varphi_{i}, \\
\gamma_{i}^{0}=\frac{\partial u_{j}}{\partial \alpha_{i}}, & \mu_{i}^{0}=\frac{\partial \varphi_{j}}{\partial \alpha_{i}}, & \mu_{i}^{2}=-\frac{4}{3 h^{2}} \frac{\partial}{\partial \alpha_{i}}\left(w_{j}+\varphi_{j}\right), & \eta_{i}^{1}=-\frac{4}{h^{2}}\left(w_{i}+\varphi_{i}\right) .
\end{aligned}
$$

Here we have introduced two new unknown functions, $w_{i}=\frac{\partial w}{\partial \alpha_{i}}$. The stress resultants are defined as

$$
\begin{aligned}
& {\left[\begin{array}{lllllllllll}
N_{i} & N_{i j} & N_{i n} & M_{i} & M_{i j} & M_{i n} & P_{i} & P_{i j} & P_{i n} & S_{i} & S_{i j}
\end{array}\right]^{T}}
\end{aligned}
$$

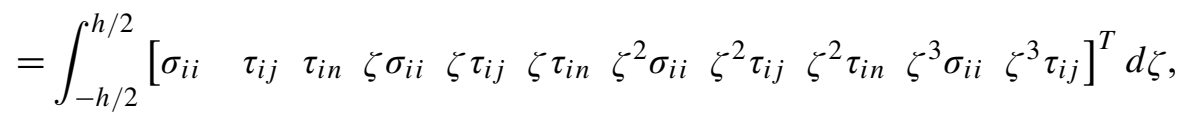

where $T$ is the transpose of a vector. The applied load per unit area of the middle surface of a plate is taken as $q=q_{1} \boldsymbol{e}_{1}+q_{2} \boldsymbol{e}_{2}-q_{n} \boldsymbol{e}_{n}$, where $\boldsymbol{e}_{1}, \boldsymbol{e}_{2}$, and $\boldsymbol{e}_{n}$ are unit vectors in the directions of the principal axes $\left(\alpha_{1}, \alpha_{2}\right)$ and thickness direction $(n)$, respectively. Let $\bar{\sigma}_{i i}, \bar{\tau}_{i j}$, and $\bar{\tau}_{i n}$ be the components of applied traction on the edges $\alpha_{i}=$ constant. The virtual work done by external loads on the plate is

$$
\delta w_{\mathrm{ext}}=\int_{\alpha_{1}} \int_{\alpha_{2}}\left(q_{1} \delta u_{1}+q_{2} \delta u_{2}-q_{n} \delta w\right) d \alpha_{1} d \alpha_{2}+\oint_{\alpha_{j}} \int_{-h / 2}^{h / 2}\left(\bar{\sigma}_{i i} \delta U_{i}+\bar{\tau}_{i j} \delta U_{j}+\bar{\tau}_{i n} \delta W\right) d \zeta d \alpha_{j}
$$

where the second integral should be taken across the boundary of the plate. Substituting (9) into (13) and (10) into (1), employing (12), setting $\sigma_{n}=0$, and carrying out the required manipulations, leads to the overall variational equation in the box on the next page.

From the first four lines of the boxed equation we derive seven governing differential equations:

$$
\begin{array}{rlrl}
\frac{\partial N_{i}}{\partial \alpha_{i}}+\frac{\partial N_{i j}}{\partial \alpha_{j}}+q_{i} & =0, & & \frac{\partial M_{i}}{\partial \alpha_{i}}+\frac{\partial M_{i j}}{\partial \alpha_{j}}-N_{i n}=0, \\
\frac{\partial N_{i n}}{\partial \alpha_{i}}+\frac{\partial N_{j n}}{\partial \alpha_{j}}-q_{n}=0, & \frac{\partial S_{i}}{\partial \alpha_{i}}+\frac{\partial S_{i j}}{\partial \alpha_{j}}-3 P_{i n}=0 .
\end{array}
$$

The remaining terms in the boxed equation lead to the boundary conditions for a plate. The boundary data on each edge $\alpha_{i}=$ constant are prescribed by selecting one member of each of the following seven 


$$
\begin{aligned}
\int_{\alpha_{1}} \int_{\alpha_{2}} & {\left[\left(\frac{\partial N_{1}}{\partial \alpha_{1}}+\frac{\partial N_{12}}{\partial \alpha_{2}}+q_{1}\right) \delta u_{1}+\left(\frac{\partial N_{2}}{\partial \alpha_{2}}+\frac{\partial N_{12}}{\partial \alpha_{1}}+q_{2}\right) \delta u_{2}+\left(\frac{\partial N_{1 n}}{\partial \alpha_{1}}+\frac{\partial N_{2 n}}{\partial \alpha_{2}}-q_{n}\right) \delta w\right.} \\
& +\left(\frac{\partial M_{1}}{\partial \alpha_{1}}-\frac{4}{3 h^{2}} \frac{\partial S_{1}}{\partial \alpha_{1}}+\frac{\partial M_{12}}{\partial \alpha_{2}}-\frac{4}{3 h^{2}} \frac{\partial S_{12}}{\partial \alpha_{2}}-N_{1 n}+\frac{4}{h^{2}} P_{1 n}\right) \delta \varphi_{1} \\
& +\left(\frac{\partial M_{2}}{\partial \alpha_{2}}-\frac{4}{3 h^{2}} \frac{\partial S_{2}}{\partial \alpha_{2}}+\frac{\partial M_{12}}{\partial \alpha_{1}}-\frac{4}{3 h^{2}} \frac{\partial S_{12}}{\partial \alpha_{1}}-N_{2 n}+\frac{4}{h^{2}} P_{2 n}\right) \delta \varphi_{2} \\
& \left.+\left(-\frac{4}{3 h^{2}} \frac{\partial S_{1}}{\partial \alpha_{1}}-\frac{4}{3 h^{2}} \frac{\partial S_{12}}{\partial \alpha_{2}}+\frac{4}{h^{2}} P_{1 n}\right) \delta w_{1}+\left(-\frac{4}{3 h^{2}} \frac{\partial S_{2}}{\partial \alpha_{2}}-\frac{4}{3 h^{2}} \frac{\partial S_{12}}{\partial \alpha_{1}}+\frac{4}{h^{2}} P_{2 n}\right) \delta w_{2}\right] d \alpha_{1} d \alpha_{2} \\
+\oint_{\alpha_{2}}[ & {\left[\bar{N}_{1}-N_{1}\right] \delta u_{1}+\left[\left(\bar{M}_{1}-\frac{4}{3 h^{2}} \bar{S}_{1}\right)-\left(M_{1}-\frac{4}{3 h^{2}} S_{1}\right)\right] \delta \varphi_{1}-\frac{4}{3 h^{2}}\left[\bar{S}_{1}-S_{1}\right] \delta w_{1}+\left[\bar{N}_{12}-N_{12}\right] \delta u_{2} } \\
& \left.+\left[\left(\bar{M}_{12}-\frac{4}{3 h^{2}} \bar{S}_{12}\right)-\left(M_{12}-\frac{4}{3 h^{2}} S_{12}\right)\right] \delta \varphi_{2}+\left[\left(\bar{N}_{1 n}+\frac{4}{3 h^{2}} \frac{\partial \bar{S}_{12}}{\partial \alpha_{2}}\right)-\left(N_{1 n}+\frac{4}{3 h^{2}} \frac{\partial S_{12}}{\partial \alpha_{2}}\right)\right] \delta w\right] d \alpha_{2} \\
+\oint_{\alpha_{1}}[ & {\left[\bar{N}_{2}-N_{2}\right] \delta u_{2}+\left[\left(\bar{M}_{2}-\frac{4}{3 h^{2}} \bar{S}_{2}\right)-\left(M_{2}-\frac{4}{3 h^{2}} S_{2}\right)\right] \delta \varphi_{2}-\frac{4}{3 h^{2}}\left[\bar{S}_{2}-S_{2}\right] \delta w_{2}+\left[\bar{N}_{12}-N_{12}\right] \delta u_{2} } \\
& \left.\left.+\left[\left(\bar{M}_{12}-\frac{4}{3 h^{2}} \bar{S}_{12}\right)-\left(M_{12}-\frac{4}{3 h^{2}} S_{12}\right)\right] \delta \varphi_{1}+\left[\left(\bar{N}_{2 n}-\frac{4}{3 h^{2}} \frac{\partial \bar{S}_{12}}{\partial \alpha_{1}}\right)-\left(N_{2 n}-\frac{4}{3 h^{2}} \frac{\partial S_{12}}{\partial \alpha_{1}}\right)\right] \delta w\right] d \alpha_{1}\right]=0 .
\end{aligned}
$$

pairs of variables as a known quantity:

$\left(N_{i}, u_{i}\right), \quad\left(M_{i}-\frac{4}{3 h^{2}} S_{i}, \varphi_{i}\right), \quad\left(S_{i}, w_{i}\right), \quad\left(N_{i j}, u_{j}\right), \quad\left(M_{i j}-\frac{4}{3 h^{2}} S_{i j}, \varphi_{j}\right), \quad\left(S_{i j}, w_{j}\right), \quad\left(N_{i n}, w\right)$,

The stress-strain relationship for a single orthotropic lamina on a plate is

$$
\left\{\begin{array}{l}
\sigma_{11} \\
\sigma_{22} \\
\tau_{2 n} \\
\tau_{1 n} \\
\tau_{12}
\end{array}\right\}=\left[\begin{array}{ccccc}
\bar{Q}_{11} & \bar{Q}_{12} & 0 & 0 & \bar{Q}_{16} \\
\bar{Q}_{12} & \bar{Q}_{22} & 0 & 0 & \bar{Q}_{26} \\
0 & 0 & \bar{Q}_{44} & \bar{Q}_{45} & 0 \\
0 & 0 & \bar{Q}_{45} & \bar{Q}_{55} & 0 \\
\bar{Q}_{16} & \bar{Q}_{26} & 0 & 0 & \bar{Q}_{66}
\end{array}\right]\left\{\begin{array}{l}
\varepsilon_{11} \\
\varepsilon_{22} \\
\gamma_{2 n} \\
\gamma_{1 n} \\
\gamma_{12}
\end{array}\right\} .
$$

The matrix components of material properties in (15) may be written in terms of the stiffness coefficients in the direction of the principal axis of material orthotropy as

$$
\begin{aligned}
& \bar{Q}_{11}=Q_{11} m^{4}+2\left(Q_{12}+2 Q_{66}\right) m^{2} n^{2}+Q_{22} n^{4}, \quad \bar{Q}_{12}=\left(Q_{11}+Q_{22}-4 Q_{66}\right) m^{2} n^{2}+Q_{12}\left(m^{4}+n^{4}\right), \\
& \bar{Q}_{22}=Q_{11} n^{4}+2\left(Q_{12}+2 Q_{66}\right) m^{2} n^{2}+Q_{22} m^{4}, \quad \bar{Q}_{45}=\left(Q_{55}-Q_{44}\right) m n, \\
& \bar{Q}_{16}=\left(Q_{11}-Q_{12}-2 Q_{66}\right) m^{3} n+\left(Q_{12}-Q_{22}+2 Q_{66}\right) m n^{3}, \\
& \bar{Q}_{26}=\left(Q_{11}-Q_{12}-2 Q_{66}\right) m n^{3}+\left(Q_{12}-Q_{22}+2 Q_{66}\right) m^{3} n, \\
& \bar{Q}_{44}=Q_{44} m^{2}+Q_{55} n^{2}, \quad \bar{Q}_{55}=Q_{44} n^{2}+Q_{55} m^{2}, \quad \bar{Q}_{66}=\left(Q_{11}+Q_{22}-2 Q_{12}\right) m^{2} n^{2}+Q_{66}\left(m^{2}-n^{2}\right)^{2} .
\end{aligned}
$$

In the equations above, $m$ and $n$ are defined as $m=\cos \chi$ and $n=\sin \chi$, where $\chi$ accounts for the angle between the principal axis of material orthotropy and the $\alpha_{1}$-axis. In fact, the principal coordinates 
of a plate differ from the material principal axes. The material principal axes make an angle $\chi$ with the principal coordinates of the plate. In terms of engineering constants, the material properties in (16) are derived as

$$
Q_{11}=\frac{E_{11}}{\Delta}, \quad Q_{12}=\frac{E_{11} \nu_{21}}{\Delta}, \quad Q_{22}=\frac{E_{22}}{\Delta}, \quad Q_{44}=G_{23}, \quad Q_{55}=G_{13}, \quad Q_{66}=G_{12},
$$

where $\Delta=1-v_{12} v_{21}$. However, if the equations in (10) are substituted into (15), the resultant equations are substituted into (12), and the integration in the thickness direction is carried out, we arrive at the following equations for the stress resultants:

$\left\{\begin{array}{c}N_{1} \\ N_{12} \\ N_{2}\end{array}\right\}=\left[\begin{array}{llll}C_{11}^{1} & C_{16}^{1} & C_{12}^{1} & C_{16}^{1} \\ C_{16}^{1} & C_{66}^{1} & C_{26}^{1} & C_{66}^{1} \\ C_{12}^{1} & C_{16}^{1} & C_{22}^{1} & C_{26}^{1}\end{array}\right]\left\{\begin{array}{l}\varepsilon_{1}^{0} \\ \gamma_{1}^{0} \\ \varepsilon_{2}^{0} \\ \gamma_{2}^{0}\end{array}\right\}+\left[\begin{array}{llll}C_{11}^{2} & C_{16}^{2} & C_{12}^{2} & C_{16}^{2} \\ C_{16}^{2} & C_{66}^{2} & C_{26}^{2} & C_{66}^{2} \\ C_{12}^{2} & C_{16}^{2} & C_{22}^{2} & C_{26}^{2}\end{array}\right]\left\{\begin{array}{l}\kappa_{1}^{0} \\ \mu_{1}^{0} \\ \kappa_{2}^{0} \\ \mu_{2}^{0}\end{array}\right\}+\left[\begin{array}{llll}C_{11}^{4} & C_{16}^{4} & C_{12}^{4} & C_{16}^{4} \\ C_{16}^{4} & C_{66}^{4} & C_{26}^{4} & C_{66}^{4} \\ C_{12}^{4} & C_{16}^{4} & C_{22}^{4} & C_{26}^{4}\end{array}\right]\left\{\begin{array}{c}\kappa_{1}^{2} \\ \mu_{1}^{2} \\ \kappa_{2}^{2} \\ \mu_{2}^{2}\end{array}\right\}$,

$\left\{\begin{array}{c}M_{1} \\ M_{12} \\ M_{2}\end{array}\right\}=\left[\begin{array}{llll}C_{11}^{2} & C_{16}^{2} & C_{12}^{2} & C_{16}^{2} \\ C_{16}^{2} & C_{66}^{2} & C_{26}^{2} & C_{66}^{2} \\ C_{12}^{2} & C_{16}^{2} & C_{22}^{2} & C_{26}^{2}\end{array}\right]\left\{\begin{array}{c}\varepsilon_{1}^{0} \\ \gamma_{1}^{0} \\ \varepsilon_{2}^{0} \\ \gamma_{2}^{0}\end{array}\right\}+\left[\begin{array}{llll}C_{11}^{3} & C_{16}^{3} & C_{12}^{3} & C_{16}^{3} \\ C_{16}^{3} & C_{66}^{3} & C_{26}^{3} & C_{66}^{3} \\ C_{12}^{3} & C_{16}^{3} & C_{22}^{3} & C_{26}^{3}\end{array}\right]\left\{\begin{array}{l}\kappa_{1}^{0} \\ \mu_{1}^{0} \\ \kappa_{2}^{0} \\ \mu_{2}^{0}\end{array}\right\}+\left[\begin{array}{llll}C_{11}^{5} & C_{16}^{5} & C_{12}^{5} & C_{16}^{5} \\ C_{16}^{5} & C_{66}^{5} & C_{26}^{5} & C_{66}^{5} \\ C_{12}^{5} & C_{16}^{5} & C_{22}^{5} & C_{26}^{5}\end{array}\right]\left\{\begin{array}{c}\kappa_{1}^{2} \\ \mu_{1}^{2} \\ \kappa_{2}^{2} \\ \mu_{2}^{2}\end{array}\right\}$,

$\left\{\begin{array}{c}P_{1} \\ P_{12} \\ P_{2}\end{array}\right\}=\left[\begin{array}{llll}C_{11}^{3} & C_{16}^{3} & C_{12}^{3} & C_{16}^{3} \\ C_{16}^{3} & C_{66}^{3} & C_{26}^{3} & C_{66}^{3} \\ C_{12}^{3} & C_{16}^{3} & C_{22}^{3} & C_{26}^{3}\end{array}\right]\left\{\begin{array}{l}\varepsilon_{1}^{0} \\ \gamma_{1}^{0} \\ \varepsilon_{2}^{0} \\ \gamma_{2}^{0}\end{array}\right\}+\left[\begin{array}{llll}C_{11}^{4} & C_{16}^{4} & C_{12}^{4} & C_{16}^{4} \\ C_{16}^{4} & C_{66}^{4} & C_{26}^{4} & C_{66}^{4} \\ C_{12}^{4} & C_{16}^{4} & C_{22}^{4} & C_{26}^{4}\end{array}\right]\left[\begin{array}{l}\kappa_{1}^{0} \\ \mu_{1}^{0} \\ \kappa_{2}^{0} \\ \mu_{2}^{0}\end{array}\right\}+\left[\begin{array}{llll}C_{11}^{6} & C_{16}^{6} & C_{12}^{6} & C_{16}^{6} \\ C_{16}^{6} & C_{66}^{6} & C_{26}^{6} & C_{66}^{6} \\ C_{12}^{6} & C_{16}^{6} & C_{22}^{6} & C_{26}^{6}\end{array}\right]\left[\begin{array}{c}\kappa_{1}^{2} \\ \mu_{1}^{2} \\ \kappa_{2}^{2} \\ \mu_{2}^{2}\end{array}\right\}$,

$\left\{\begin{array}{c}S_{1} \\ S_{12} \\ S_{2}\end{array}\right\}=\left[\begin{array}{llll}C_{11}^{4} & C_{16}^{4} & C_{12}^{4} & C_{16}^{4} \\ C_{16}^{4} & C_{66}^{4} & C_{26}^{4} & C_{66}^{4} \\ C_{12}^{4} & C_{16}^{4} & C_{22}^{4} & C_{26}^{4}\end{array}\right]\left\{\begin{array}{c}\varepsilon_{1}^{0} \\ \gamma_{1}^{0} \\ \varepsilon_{2}^{0} \\ \gamma_{2}^{0}\end{array}\right\}+\left[\begin{array}{llll}C_{11}^{5} & C_{16}^{5} & C_{12}^{5} & C_{16}^{5} \\ C_{16}^{5} & C_{66}^{5} & C_{26}^{5} & C_{66}^{5} \\ C_{12}^{5} & C_{16}^{5} & C_{22}^{5} & C_{26}^{5}\end{array}\right]\left\{\begin{array}{l}\kappa_{1}^{0} \\ \mu_{1}^{0} \\ \kappa_{2}^{0} \\ \mu_{2}^{0}\end{array}\right\}+\left[\begin{array}{llll}C_{11}^{7} & C_{16}^{7} & C_{12}^{7} & C_{16}^{7} \\ C_{16}^{7} & C_{66}^{7} & C_{26}^{7} & C_{66}^{7} \\ C_{12}^{7} & C_{16}^{7} & C_{22}^{7} & C_{26}^{7}\end{array}\right]\left\{\begin{array}{c}\kappa_{1}^{2} \\ \mu_{1}^{2} \\ \kappa_{2}^{2} \\ \mu_{2}^{2}\end{array}\right\}$,

$$
\begin{aligned}
& \left\{\begin{array}{l}
N_{1 n} \\
N_{2 n}
\end{array}\right\}=\left[\begin{array}{ll}
C_{55}^{1} & C_{54}^{1} \\
C_{45}^{1} & C_{44}^{1}
\end{array}\right]\left\{\begin{array}{l}
\lambda_{1}^{0} \\
\lambda_{2}^{0}
\end{array}\right\}+\left[\begin{array}{ll}
C_{55}^{3} & C_{54}^{3} \\
C_{45}^{3} & C_{44}^{3}
\end{array}\right]\left\{\begin{array}{l}
\eta_{1}^{1} \\
\eta_{2}^{1}
\end{array}\right\}, \\
& \left\{\begin{array}{l}
M_{1 n} \\
M_{2 n}
\end{array}\right\}=\left[\begin{array}{ll}
C_{55}^{2} & C_{54}^{2} \\
C_{45}^{2} & C_{44}^{2}
\end{array}\right]\left\{\begin{array}{l}
\lambda_{1}^{0} \\
\lambda_{2}^{0}
\end{array}\right\}+\left[\begin{array}{ll}
C_{55}^{4} & C_{54}^{4} \\
C_{45}^{4} & C_{44}^{4}
\end{array}\right]\left\{\begin{array}{l}
\eta_{1}^{1} \\
\eta_{2}^{1}
\end{array}\right\}, \\
& \left\{\begin{array}{l}
P_{1 n} \\
P_{2 n}
\end{array}\right\}=\left[\begin{array}{ll}
C_{55}^{3} & C_{54}^{3} \\
C_{45}^{3} & C_{44}^{3}
\end{array}\right]\left\{\begin{array}{l}
\lambda_{1}^{0} \\
\lambda_{2}^{0}
\end{array}\right\}+\left[\begin{array}{ll}
C_{55}^{5} & C_{54}^{5} \\
C_{45}^{5} & C_{44}^{5}
\end{array}\right]\left\{\begin{array}{l}
\eta_{1}^{1} \\
\eta_{2}^{1}
\end{array}\right\},
\end{aligned}
$$

where

$$
C_{i j}^{p}=\frac{1}{p} \sum_{k=1}^{N}\left(\bar{Q}_{i j}\right)_{k}\left(h_{k}^{p}-h_{k-1}^{p}\right), \quad p \in\{1,2, \ldots, 7\},
$$


and $h_{k}-h_{k-1}$ is the thickness of the $k$-th layer. Substitution of (11) into the equations on page 1776 yields the stress resultants in terms of the displacement components. These equations, combined with (14), form a system of 25 first-order partial differential equations for displacements and stress resultants: $[K]\left\{u_{1}, u_{2}, w, \varphi_{1}, \varphi_{2}, w_{1}, w_{2}, N_{1}, N_{12}, N_{2}, M_{1}, M_{12}, M_{2}\right.$,

$$
\left.P_{1}, P_{12}, P_{2}, S_{1}, S_{12}, S_{2}, N_{1 n}, N_{2 n}, M_{1 n}, M_{2 n}, P_{1 n}, P_{2 n}\right\}^{T}=\left\{-q_{1},-q_{2}, q_{n}, 0, \ldots, 0\right\}^{T} \text {. }
$$

The coefficients of the matrix $K$ are given in the box below (19). A numerical solution for (19) can be achieved by means of the GDQ method. The method is detailed, for example, in [Shu 1991; Bert et al. 1988], and its application to first-order differential equations is summarized here. In the GDQ method,

\section{Nonzero entries of $K$ referenced by row and column}

Rows 1 to 7 :
$1,8: \frac{\partial}{\partial \alpha_{1}}$
$1,9: \frac{\partial}{\partial \alpha_{2}}$
$2,9: \frac{\partial}{\partial \alpha_{1}}$
$2,10: \frac{\partial}{\partial \alpha_{2}}$
$3,20: \frac{\partial}{\partial \alpha_{1}}$
$3,21: \frac{\partial}{\partial \alpha_{2}}$
4,11: $\frac{\partial}{\partial \alpha_{1}}$
4,12: $\frac{\partial}{\partial \alpha_{2}}$
$4,20:-1$
5,13: $\frac{\partial}{\partial \alpha_{2}}$
5,12: $\frac{\partial}{\partial \alpha_{1}}$
$5,21:-1$
$6,17: \frac{\partial}{\partial \alpha_{1}}$
$6,18: \frac{\partial}{\partial \alpha_{2}}$
$6,24:-3$
7,19: $\frac{\partial}{\partial \alpha_{2}}$
$7,18: \frac{\partial}{\partial \alpha_{1}}$
$7,25:-3$

Rows 8 to 19 :

$$
k, k:-1
$$

$k, 1: A_{1}^{\prime} \frac{\partial}{\partial \alpha_{1}}+C_{1}^{\prime} \frac{\partial}{\partial \alpha_{2}} \quad k, 4:\left(A_{2}^{\prime}-\frac{4}{3 h^{2}} A_{4}^{\prime}\right) \frac{\partial}{\partial \alpha_{1}}+\left(C_{2}^{\prime}-\frac{4}{3 h^{2}} C_{4}^{\prime}\right) \frac{\partial}{\partial \alpha_{2}} \quad k, 6:-\frac{4}{3 h^{2}} A_{4}^{\prime} \frac{\partial}{\partial \alpha_{1}}-\frac{4}{3 h^{2}} C_{4}^{\prime} \frac{\partial}{\partial \alpha_{2}}$

$k, 2: B_{1}^{\prime} \frac{\partial}{\partial \alpha_{2}}+C_{1}^{\prime} \frac{\partial}{\partial \alpha_{1}} \quad k, 5:\left(B_{2}^{\prime}-\frac{4}{3 h^{2}} B_{4}^{\prime}\right) \frac{\partial}{\partial \alpha_{2}}+\left(C_{2}^{\prime}-\frac{4}{3 h^{2}} C_{4}^{\prime}\right) \frac{\partial}{\partial \alpha_{1}} \quad k, 7:-\frac{4}{3 h^{2}} B_{4}^{\prime} \frac{\partial}{\partial \alpha_{1}}-\frac{4}{3 h^{2}} C_{4}^{\prime} \frac{\partial}{\partial \alpha_{2}}$ where
for $k \in\{8,11,14,17\}$,
$n=\frac{1}{3}(k-8)$,
$A_{l}^{\prime}=C_{11}^{n+l}$,
$B_{l}^{\prime}=C_{12}^{n+l}$,
$C_{l}^{\prime}=C_{16}^{n+l} ;$
for $k \in\{9,12,15,18\}$,
$n=\frac{1}{3}(k-9)$,
$A_{l}^{\prime}=C_{16}^{n+l}$,
$B_{l}^{\prime}=C_{26}^{n+l}$,
$C_{l}^{\prime}=C_{66}^{n+l}$;
for $k \in\{10,13,16,19\}$,
$n=\frac{1}{3}(k-10)$,
$A_{l}^{\prime}=C_{12}^{n+l}$,
$B_{l}^{\prime}=C_{22}^{n+l}$,
$C_{l}^{\prime}=C_{16}^{n+l}$,

Rows 20 to 25 :
$k, k:=-1$
$k, 4:=A_{1}^{\prime}-\frac{4}{h^{2}} A_{3}^{\prime}$
$k, 6:=-\frac{4}{h^{2}} A_{3}^{\prime}$
$k, 3:=A_{1}^{\prime} \frac{\partial}{\partial \alpha_{1}}+B_{1}^{\prime} \frac{\partial}{\partial \alpha_{2}}$
$k, 5:=B_{1}^{\prime}-\frac{4}{h^{2}} B_{3}^{\prime}$
$k, 7:=-\frac{4}{3 h^{2}} A_{3}^{\prime}$

where
for $k \in\{20,22,24\}$,
$n=\frac{1}{2}(k-20)$,
$A_{l}^{\prime}=C_{55}^{n+l}$,
$B_{l}^{\prime}=C_{45}^{n+l}$,
for $k \in\{21,23,25\}$,
$n=\frac{1}{2}(k-21)$,
$A_{l}^{\prime}=C_{45}^{n+l}$,
$B_{l}^{\prime}=C_{44}^{n+l}$. 
the derivative of a function at any discrete point in a given direction is approximated by the weighted linear sum of the function values at all sampling points in that direction,

$$
\frac{d F\left(x_{k}\right)}{d x}=\sum_{l=1}^{N} C_{k l} F\left(x_{l}\right), \quad k \in\{1,2, \ldots, N\}
$$

where $N$ denotes the number of sampling points selected in the $x$-direction and $C_{k l}$ are the weighting coefficients of the first derivative with respect to the variable $x$. Taking the Lagrange interpolation polynomials as test functions, the coefficients in (20) result in

$$
\begin{array}{rlrl}
C_{k l} & =\frac{M\left(x_{k}\right)}{\left(x_{k}-x_{l}\right) M\left(x_{l}\right)}, & k, l=1,2, \ldots, N, \quad k \neq l, \\
C_{k k} & =-\sum_{\substack{l=1 \\
l \neq k}}^{N} C_{k l}, & k & =1,2, \ldots, N,
\end{array}
$$

where $M\left(x_{k}\right)=\prod_{\substack{l=1 \\ l \neq k}}^{N}\left(x_{k}-x_{l}\right)$. The sampling points are chosen in the form of a cosine distribution as

$$
x_{k}=\frac{a}{2}\left[1-\cos \left(\frac{k-1}{N-1} \pi\right)\right], \quad k=1,2, \ldots, N,
$$

where $a$ is the length in $x$-direction. Differentiating (19) with respect to $\alpha_{1}$ and $\alpha_{2}$ and using (20) together with the boundary conditions (see page 1775) leads to an overdetermined system of algebraic equations for displacements and stress resultants at the sampling points. These equations are then solved using least-squares minimization methods.

\section{Numerical results}

To verify the methodology developed in this study, the bending of symmetric ([0/90/0] and [90/0/90]) and antisymmetric [0/90] cross ply square plates, subjected to uniformly distributed transverse loads, were considered. A material with the following properties was used in these numerical calculations:

$$
\frac{E_{1}}{E_{2}}=25, \quad \frac{G_{12}}{E_{2}}=\frac{G_{13}}{E_{2}}=0.5, \quad \frac{G_{23}}{E_{2}}=0.2, \quad v_{12}=0.25,
$$

in which $E_{1}$ and $E_{2}$ are the in-plane Young's modulus in the $\alpha_{1}$ and $\alpha_{2}$ coordinate directions. $G_{12}$ is the in-plane shear modulus, $G_{13}$ and $G_{23}$ are the transverse shear modulus in the $\alpha_{1}-n$ and $\alpha_{2}-n$ planes, respectively, whereas $v_{12}$ is the major Poisson's ratio in the $\alpha_{1}-\alpha_{2}$ plane. The quantities $w^{*}$ and $M_{1}^{*}$ are

$$
w^{*}=-\frac{10^{3} E_{2} h^{3}}{q_{n} a^{4}} w, \quad M_{1}^{*}=\frac{10^{3} M_{i}}{q_{n} a^{2}} .
$$

Here, ' $a$ ' is the length of the edges of the square, and $q_{n}$ denotes the uniformly distributed transverse load. In this study, three models with various boundary conditions were considered. The first model, called (S-S-S-S), is a plate with an SS2-type simply supported boundary condition on all edges. The second model, called (S-S-S-SF), is a plate with SS2-type simply supported boundary condition on three edges, and the fourth edge, corresponding to the edge $\alpha_{1}=a$, is a mixed boundary condition of free and 


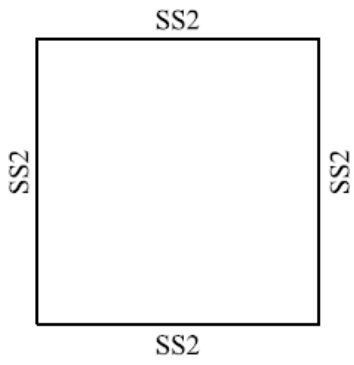

(S-S-S-S)

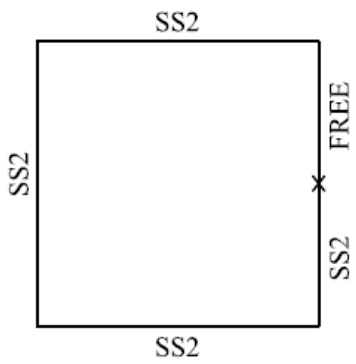

(S-S-S-SF)

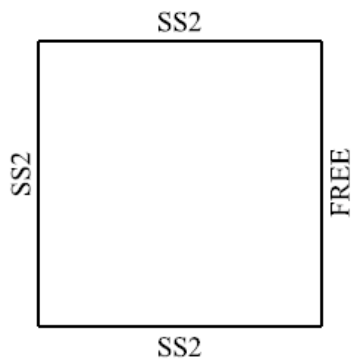

(S-S-S-F)

Figure 1. The models used in the present study with their various boundary conditions.

SS2-type simply supported boundary conditions, as shown in Figure 1. (S-S-S-F) is the last model and has three edges subjected to the SS2 boundary condition, and the edge corresponding to the edge $\alpha_{1}=a$ is free.

The SS2-type simply supported boundary conditions and free edge defined on the edge $\alpha_{i}=$ constant are as follows:

SS2 type: $\quad u_{i}=0, \quad M_{i}=0, \quad S_{i}=0, \quad N_{i j}=0, \quad \varphi_{j}=0, \quad w_{j}=0, \quad w=0$.

Free edge: $\quad N_{i}=0, \quad M_{i}=0, \quad S_{i}=0, \quad N_{i j}=0, \quad M_{i j}=0, \quad S_{i j}=0, \quad N_{i n}=0$.

In all models, the displacements and moments are computed at the center of the plate. Because the bending of a plate with mixed boundary conditions has not been extensively investigated, we compared the models to simulations performed with the ANSYS version 5.4 finite element software. The finite element mesh is composed of 400 SHELL99 elements with identical dimensions.

Table 1 compares the normalized central deflections of antisymmetric [0/90] and symmetric [0/90/0] and [90/0/90] cross ply square plates, characterized by four different $a / h$ ratios, for the three models, computed using our approach versus ANSYS. In each direction, fifteen grid points are used for model (S-S-S-S), 17 for model (S-S-S-SF), and 19 for model (S-S-S-F). Table 2 shows the analogous compares for central moments.

The maximum $w^{*}$ discrepancy between the two sets of results is observed for the (S-S-S-SF) model with a $[0 / 90 / 0]$ lamination and $a / h=10$. In comparing the results with FE, we mention that the boundary conditions used in the present approach are not identical to those in ANSYS. Therefore, we expect some discrepancies between the results. Nonetheless, discrepancies should not be more than $11 \%$.

The discrepancies of the normalized central moments, $M_{1}^{*}$, between the two sets of results can be attributed to the fact that ANSYS calculates moments on grid points by extrapolating the moment calculated over the Gaussian points. Therefore, this method produces some approximations in the calculation of moments on grid points.

In the [90/0/90] lamination, the normalized central deflections of (S-S-S-SF) are larger than those of $(\mathrm{S}-\mathrm{S}-\mathrm{S}-\mathrm{F})$. A finite element analysis with 1600 elements $(a / h=10)$ was performed. The value of $w^{*}$, in the center of both (S-S-S-SF) and (S-S-S-F) models, is 11.73.

The first three rows of Figure 2 display the convergence (with $n_{1}=n_{2}=n$ ) of normalized transverse displacements $w^{*}$ and normalized moments $M_{1}^{*}$ for the (S-S-S-S), (S-S-S-SF), and (S-S-S-F) models 

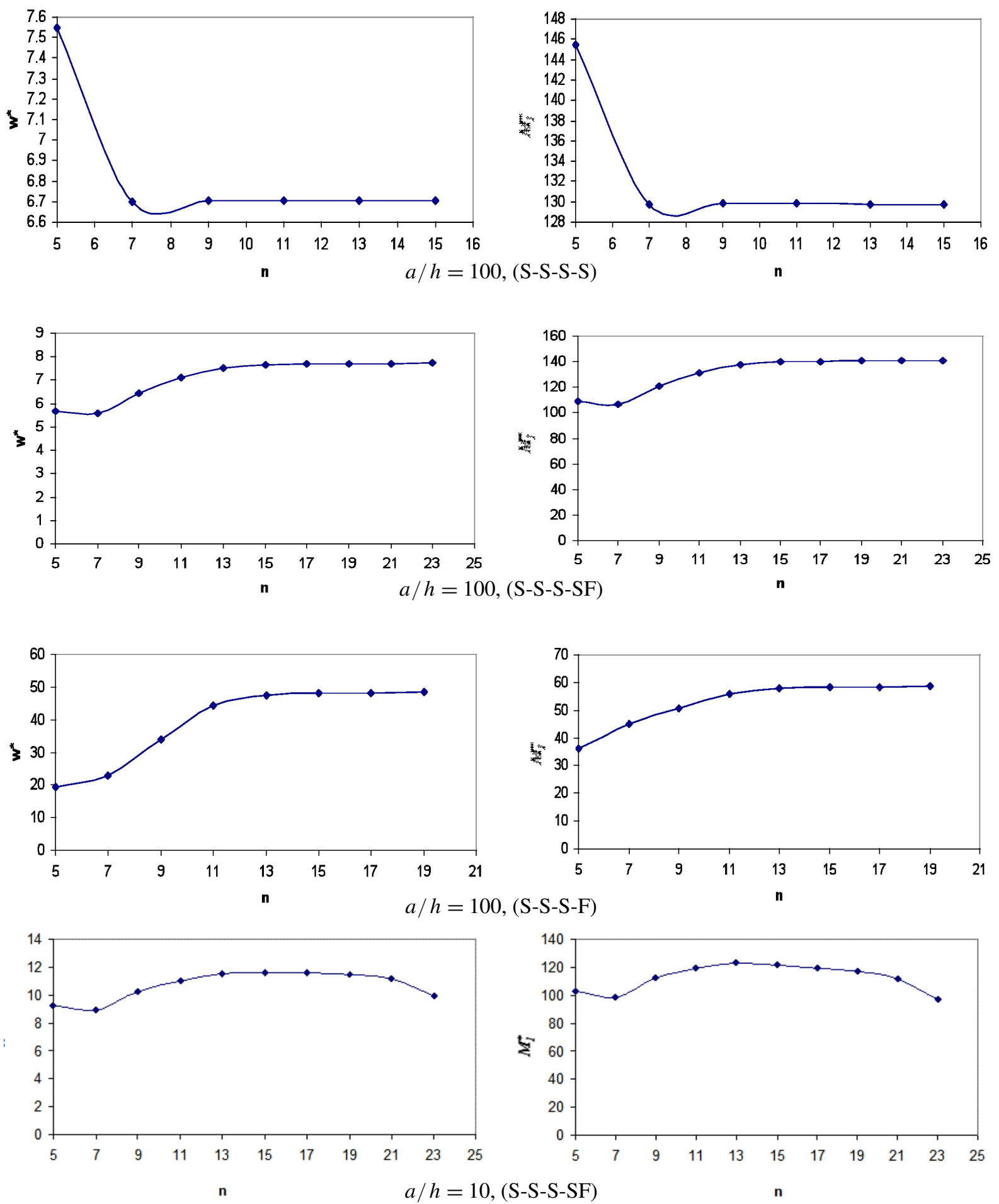

Figure 2. Convergence of the normalized central deflection $w^{*}$ (left) and the normalized central moment $M_{1}^{*}$ (right) of a symmetric cross ply [0/90/0] for the three models. 


\begin{tabular}{|c|c|c|c|c|c|c|c|c|c|}
\hline \multirow[b]{2}{*}{$a / h$} & & \multicolumn{2}{|c|}{$(\mathrm{S}-\mathrm{S}-\mathrm{S}-\mathrm{S})$} & \multicolumn{3}{|c|}{ (S-S-S-SF) } & \multicolumn{3}{|c|}{$(\mathrm{S}-\mathrm{S}-\mathrm{S}-\mathrm{F})$} \\
\hline & & [0/90] & {$[0 / 90 / 0]$} & {$[0 / 90]$} & {$[0 / 90 / 0]$} & {$[90 / 0 / 90]$} & [0/90] & {$[0 / 90 / 0]$} & [90/0/90] \\
\hline \multirow{2}{*}{10} & present & 11.73 & 10.98 & 11.65 & 11.60 & 11.41 & 17.88 & 52.79 & 10.97 \\
\hline & ANSYS & 11.63 & 11.70 & 12.72 & 13.10 & 11.72 & 17.73 & 53.44 & 11.73 \\
\hline \multirow{2}{*}{20} & present & 9.82 & 7.79 & 10.40 & 8.65 & 7.80 & 15.12 & 49.54 & 7.67 \\
\hline & ANSYS & 9.72 & 7.96 & 10.58 & 9.34 & 7.96 & 14.98 & 49.64 & 7.85 \\
\hline \multirow{2}{*}{50} & present & 9.23 & 6.84 & 9.76 & 7.87 & 6.84 & 14.27 & 48.54 & 6.72 \\
\hline & ANSYS & 9.18 & 6.86 & 9.96 & 8.06 & 6.86 & 14.20 & 48.51 & 6.74 \\
\hline \multirow{2}{*}{100} & present & 9.14 & 6.71 & 9.68 & 7.72 & 6.70 & 14.10 & 48.40 & 6.58 \\
\hline & ANSYS & 9.11 & 6.73 & 9.87 & 7.83 & 6.73 & 14.09 & 48.55 & 6.61 \\
\hline
\end{tabular}

Table 1. Normalized central deflections of square plates with different $a / h$ ratios, cross ply patterns and models, calculated under the present approach and with ANSYS.

\begin{tabular}{|c|c|c|c|c|c|c|c|c|c|}
\hline \multirow[b]{2}{*}{$a / h$} & & \multicolumn{2}{|c|}{$(\mathrm{S}-\mathrm{S}-\mathrm{S}-\mathrm{S})$} & \multicolumn{3}{|c|}{ (S-S-S-SF) } & \multicolumn{3}{|c|}{$(\mathrm{S}-\mathrm{S}-\mathrm{S}-\mathrm{F})$} \\
\hline & & [0/90] & {$[0 / 90 / 0]$} & {$[0 / 90]$} & {$[0 / 90 / 0]$} & {$[90 / 0 / 90]$} & {$[0 / 90]$} & {$[0 / 90 / 0]$} & [90/0/90] \\
\hline \multirow{2}{*}{10} & present & 69.42 & 122.88 & 63.19 & 116.55 & 14.94 & 23.14 & 53.90 & 7.26 \\
\hline & ANSYS & 69.96 & 120.97 & 68.47 & 125.10 & 13.94 & 22.81 & 53.32 & 7.65 \\
\hline \multirow{2}{*}{20} & present & 70.30 & 128.06 & 70.07 & 136.87 & 9.82 & 22.73 & 57.01 & 5.50 \\
\hline & ANSYS & 70.67 & 128.20 & 69.08 & 141.03 & 9.80 & 22.66 & 56.89 & 5.61 \\
\hline \multirow{2}{*}{50} & present & 70.64 & 129.57 & 73.75 & 140.15 & 8.58 & 22.57 & 58.21 & 4.92 \\
\hline & ANSYS & 70.92 & 129.93 & 69.19 & 141.90 & 8.56 & 22.63 & 58.42 & 4.94 \\
\hline \multirow{2}{*}{100} & present & 70.70 & 129.78 & 72.57 & 140.85 & 8.47 & 22.59 & 58.61 & 4.84 \\
\hline & ANSYS & 70.97 & 130.17 & 69.18 & 142.02 & 8.39 & 22.63 & 58.82 & 4.84 \\
\hline
\end{tabular}

Table 2. Normalized central moments of square plates with different $a / h$ ratios, cross ply patterns and models, calculated under the present approach and with ANSYS.

describing symmetric cross ply [0/90/0] square plates with $a / h=100$. Rapid convergence was observed for the (S-S-S-S) model, and convergence of the (S-S-S-SF) model was the slowest. The convergence of each model was similar for different $a / h$ ratios, with the exception of model (S-S-S-SF), with $a / h=10$, shown at the bottom in Figure 2. It is interesting to note that, as observed in this latter figure, the curve of convergence initially ascends, then descends at $n=15$. The number 15 is reported as the convergence number for this case. Actually, convergence for the deflections and moments, for thicker plates, was accomplished with a smaller number of grid points. This characteristic was observed by Lanhe et al. [2005] in the vibration analysis of composite plates, by applying a least square GDQ method based on FSDT.

Figure 3, top left, compares the variation in central deflections $w^{*}$ for three different laminations, including antisymmetric [0/90] and symmetric [0/90/0], [90/0/90], with respect to the $a / h$ ratio, for the (S-S-S-S) model. It is seen in the figure that the normalized central deflections of two symmetric cross ply laminates are equal because the boundary conditions imposed on all edges are similar. However, the normalized central deflections, of the antisymmetric cross ply plate are considerably larger that their 

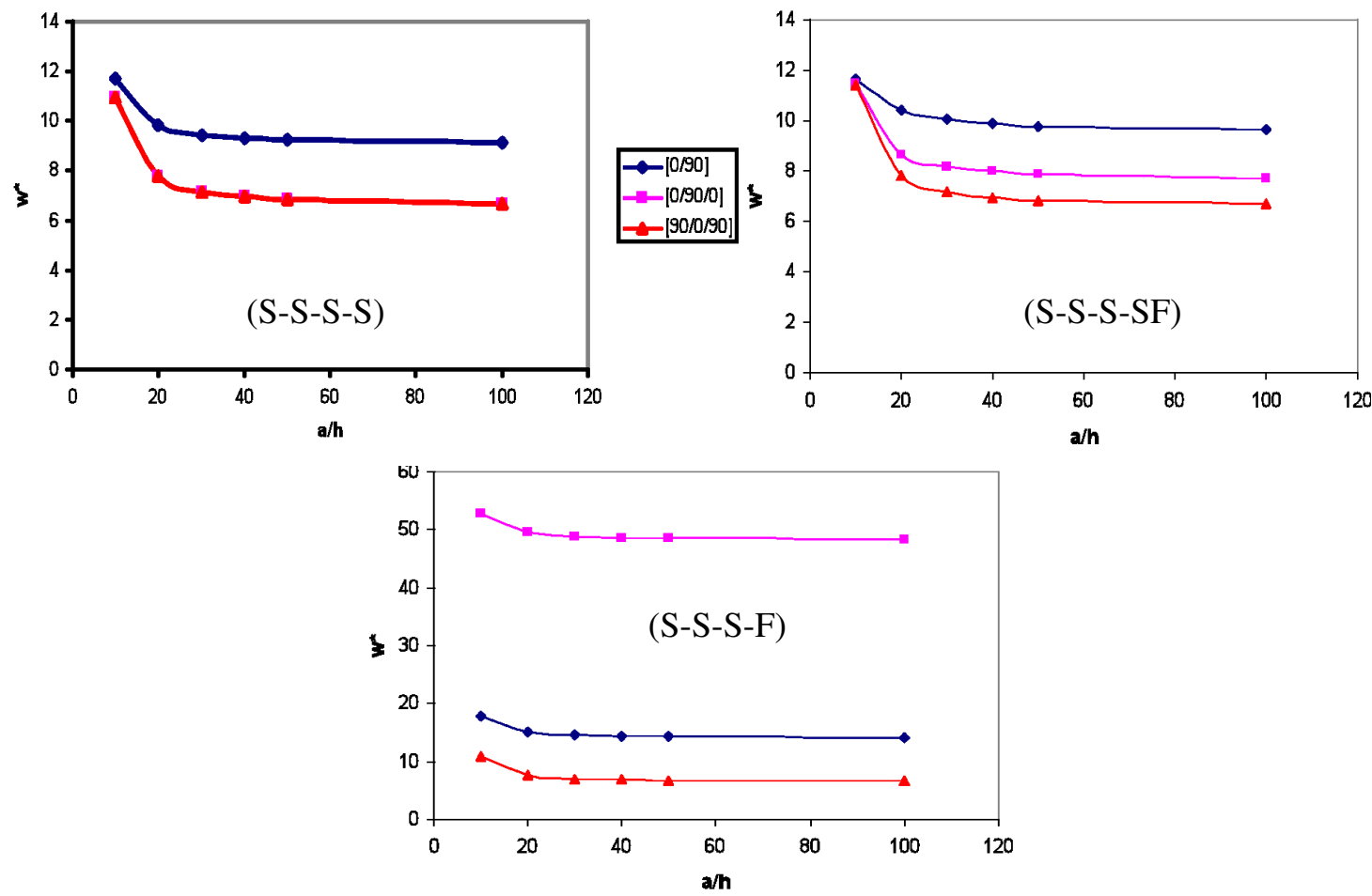

Figure 3. Variation in central deflection $w^{*}$ with respect to the $a / h$ ratio for [0/90], [0/90/0], and [90/0/90] laminations, in the three models.

symmetric counterparts. As the $a / h$ ratio decreases from thin to moderately thick, the difference between symmetric and antisymmetric lamination decreases, and this decrease is much larger for moderately thick aspect ratios $(10<a / h<20)$. This behavior is known as the "beam-column/tie bar" effect caused by the bending-stretching coupling present in the antisymmetric laminate.

Figure 3, top right, presents a comparison of the variation in central deflections $w^{*}$ for three different laminations, including antisymmetric [0/90] and symmetric [0/90/0], as well as [90/0/90], with respect to the $a / h$ ratio, for the (S-S-S-SF) model. As can be observed from the rule, the normalized central deflections of the two symmetric cross ply laminates are different, because the boundary conditions on the two edges $\alpha_{1}=0$ and $\alpha_{1}=a$ are different. Therefore, in the [0/90/0] lamination, the orientation of most fibers of the laminate material is along to $\alpha_{1}$ coordinate. This increases the deflection caused by the free part of the edge $\alpha_{1}=a$. The difference between the behavior of the laminations [0/90/0] and [90/0/90] for the (S-S-S-SF) model is shown in the first two panels of Figure 4.

Figure 3, bottom, shows the comparison of the of the variation in the central deflections $w^{*}$ for three different laminations, including antisymmetric [0/90] and symmetric [0/90/0], [90/0/90], with respect to the $a / h$ ratio, for the (S-S-S-F) model. The figure highlights the differences between the laminations. In the $[0 / 90 / 0]$ lamination, as was described for (S-S-S-SF), the direction of most fibers of the laminate material is along to $\alpha_{1}$ axis. This contributes to an increase in the deflection of the plate. The difference between the behavior of laminations [0/90/0] and [90/0/90] is shown in Figure 4, right. 

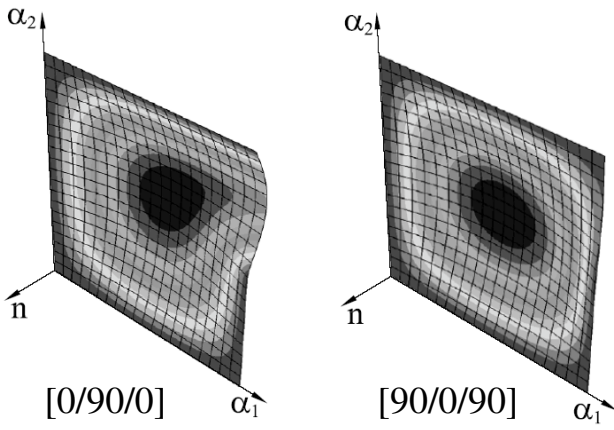

$(\mathrm{S}-\mathrm{S}-\mathrm{S}-\mathrm{SF})$
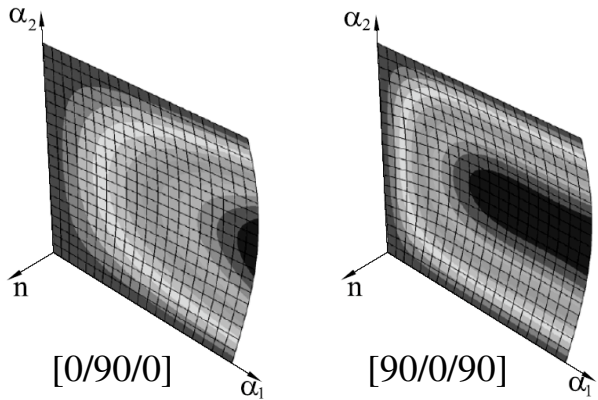

$(\mathrm{S}-\mathrm{S}-\mathrm{S}-\mathrm{F})$

Figure 4. Displacement contours of a plate with laminations $[0 / 90 / 0]$ and $[90 / 0 / 90]$ for two of the models.

Figure 5 compares the central deflection of the (S-S-S-S) and (S-S-S-SF) models, for [0/90], [0/90/0], and [90/0/90] laminations. The maximum difference between (S-S-S-S) and (S-S-S-SF) is observed in the [0/90/0] lamination, and the minimum difference is observed in the [90/0/90] lamination. This effect can be understood from the explanation given for Figure 4, left.
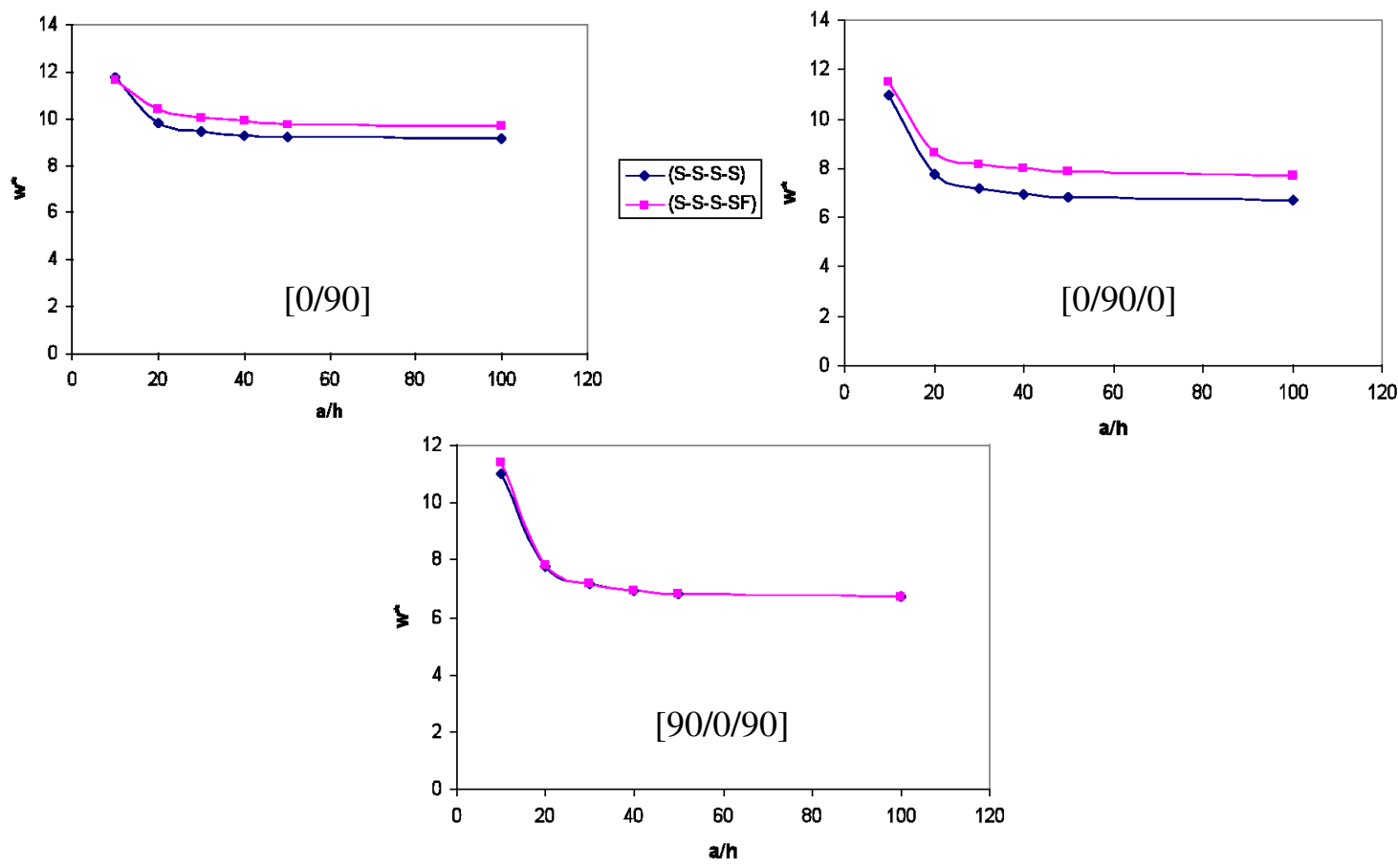

Figure 5. Variation in central deflection $w^{*}$ with respect to the $a / h$ ratio for $[0 / 90]$, [0/90/0], and [90/0/90] laminations, in the (S-S-S-S) and (S-S-S-SF) models. 


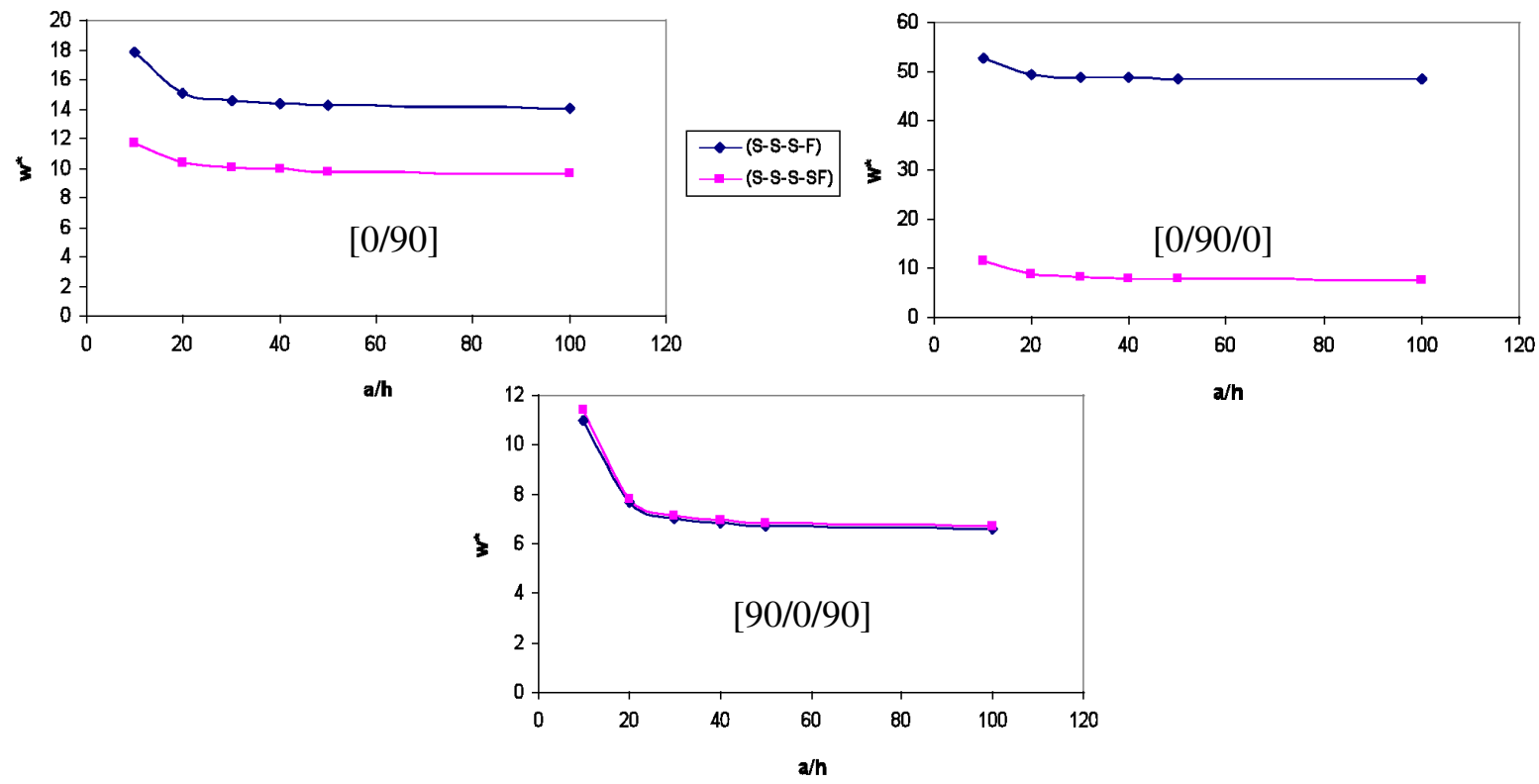

Figure 6. Variation in central deflection $w^{*}$ with respect to the $a / h$ ratio for [0/90], [0/90/0], and [90/0/90] laminations, in the (S-S-S-F) and (S-S-S-SF) models.

Figure 6 illustrates the comparison of central deflections, $w^{*}$, of the (S-S-S-SF) and (S-S-S-F) models for [0/90], [0/90/0], and [90/0/90] laminations. The maximum difference between (S-S-S-S) and (S-S$\mathrm{S}-\mathrm{SF}$ ) is observed in the [0/90/0] lamination, and the minimum difference is in the [90/0/90] lamination. Although the boundary condition, SF, is much stronger than the boundary condition, F, in the [90/0/90] lamination, most of the normalized central deflections in the model (S-S-S-SF) are larger than those of (S$\mathrm{S}-\mathrm{S}-\mathrm{F}$ ). In the case of an isotropic material, it is always expected that the normalized central deflections of (S-S-S-F) are larger than those of (S-S-S-SF). However, when using composite materials, the behavior of the structure is more complex. We come to the conclusion that, in addition to the boundary conditions, the direction of the lamination contributes to the behavior of the structure.

\section{Conclusion}

A new formulation, based on higher-order shear deformation theory, is presented to solve the problem of laminated plates with mixed boundary conditions. Using the principle of virtual work, the governing equations, together with the required boundary conditions for higher-order shear deformation, are formulated. The equations are solved numerically by means of the generalized differential quadrature method. Three models differing in their boundary conditions on one edge are considered. The results are compared against solutions obtained using ANSYS, and reasonable agreement is observed. Numerical results presented here for cross ply plates demonstrate a reasonably fast convergence for the (S-S-S-S) model and slow convergence for the (S-S-S-SF) model.

When an isotropic material is used, it is usually expected that, because the constraint on SF is much stronger than the constraint on F, the normalized central deflections of (S-S-S-F) are larger than those of (S-S-S-SF). According to the tables, for the lamination [90/0/90], normalized central deflections of 
(S-S-S-SF) are larger than those of (S-S-S-F). This result illustrates that when a composite material is used, the behavior of the structure is more complex, and we can come to the conclusion that, in addition to the boundary conditions, the direction of the lamination contributes to the behavior of the structures.

The direction of fibers in the [0/90/0] and [90/0/90] laminations has a significant effect, especially for models (S-S-S-SF) and (S-S-S-F). When the direction of most of the fibers is perpendicular to the free edge, the central deflections of the plates are larger than when it's parallel. The effect of the transverse shear deformation is described by the conceptual bending-stretching coupling effect, a characteristic of antisymmetric laminates. This characteristic is obvious in the model (S-S-S-S).

\section{References}

[Aghdam et al. 2006] M. M. Aghdam, M. R. N. Farahani, M. Dashty, and S. M. Rezaei Niya, "Application of generalized differential quadrature method to the bending of thick laminated plates with various boundary conditions", Appl. Mech. Mater. 5-6 (2006), 407-414.

[Basset 1890] A. B. Basset, "On the extension and flexure of cylindrical and spherical thin elastic shells", Phil. Trans. R. Soc. A 181 (1890), 433-480.

[Bellman et al. 1972] R. Bellman, B. G. Kashef, and J. Casti, "Differential quadrature: a technique for the rapid solution of nonlinear partial differential equations", J. Comput. Phys. 10:1 (1972), 40-52.

[Bert and Chen 1978] C. W. Bert and T. L. C. Chen, "Effect of shear deformation on vibration of antisymmetric angle-ply laminated rectangular plates", Int. J. Solids Struct. 14:6 (1978), 465-473.

[Bert and Francis 1974] C. W. Bert and P. H. Francis, "Composite material mechanics: structural mechanics", AIAA J. 12:9 (1974), 1173-1186.

[Bert et al. 1988] C. W. Bert, S. K. Jang, and A. G. Striz, "Two new approximate methods for analyzing free vibration of structural components", AIAA J. 26:5 (1988), 612-618.

[Bert et al. 1989] C. W. Bert, S. K. Jang, and A. G. Striz, "Nonlinear bending analysis of orthotropic rectangular plates by the method of differential quadrature", Comput. Mech. 5:2-3 (1989), 217-226.

[Lanhe et al. 2005] W. Lanhe, L. Hua, and D. Wang, "Vibration analysis of generally laminated composite plates by the moving least squares differential quadrature method”, Compos. Struct. 68:3 (2005), 319-330.

[Li and Cheng 2005] J.-J. Li and C.-J. Cheng, "Differential quadrature method for nonlinear vibration of orthotropic plates with finite deformation and transverse shear effect", J. Sound Vib. 281:1-2 (2005), 295-309.

[Malekzadeh and Setoodeh 2007] P. Malekzadeh and A. R. Setoodeh, "Large deformation analysis of moderately thick laminated plates on nonlinear elastic foundations by DQM”, Compos. Struct. 80:4 (2007), 569-579.

[Mindlin 1951] R. D. Mindlin, "Influence of rotatory inertia and shear on flexural motions of isotropic, elastic plates", J. Appl. Mech. (ASME) 18:1 (1951), 31-38.

[Naghdi 1956] P. M. Naghdi, “A survey of recent progress in theory of elastic shells", Appl. Mech. Rev. (ASME) 9:9 (1956), 365-388.

[Quan and Chang 1989] J. R. Quan and C. T. Chang, "New insights in solving distributed system equations by the quadrature methods, I: Analysis”, Comput. Chem. Eng. 13:7 (1989), 779-788.

[Reddy and Liu 1985] J. N. Reddy and C. F. Liu, "A higher-order shear deformation theory of laminated elastic shells", Int. J. Eng. Sci. 23:3 (1985), 319-330.

[Reissner 1945] E. Reissner, "The effect of transverse shear deformation on the bending of elastic plates", J. Appl. Mech. (ASME) 12:2 (1945), 69-77.

[Sherbourne and Pandey 1991] A. N. Sherbourne and M. D. Pandey, "Differential quadrature method in the buckling analysis of beams and composite plates", Comput. Struct. 40:4 (1991), 903-913.

[Shu 1991] C. Shu, Generalized differential quadrature integral quadrature and application to the simulation of incompressible viscous flows including parallel computation, Ph.D. thesis, University of Glasgow, 1991. 
[Shu and Richards 1990] C. Shu and B. E. Richards, "High resolution of natural convection in a square cavity by generalized differential quadrature", pp. 978-985 in Numerical methods in engineering: theory and appreciations (NUMETA 90) (Swansea, 1990), vol. 2, edited by G. N. Pande and J. Middleton, Elsevier, London, 1990.

[Striz et al. 1988] A. G. Striz, S. K. Jang, and C. W. Bert, "Nonlinear bending analysis of thin circular plates by differential quadrature", Thin-Walled Struct. 6:1 (1988), 51-62.

[Tornabene and Viola 2008] F. Tornabene and E. Viola, "2-D solution for free vibrations of parabolic shells using generalized differential quadrature method", Eur. J. Mech. A Solids 27:6 (2008), 1001-1025.

Received 27 Feb 2009. Revised 9 Apr 2009. Accepted 23 Jun 2009.

Mojgan YaghoubShahi: moyaeng@gmail.com

Department of Civil and Environmental Engineering, Amirkabir University of Technology, 424 Hafez Avenue, Tehran 15914, Iran

HOSSEIN RAJAIE: rajaieh@aut.ac.ir

Department of Civil and Environmental Engineering, Amirkabir University of Technology, 424 Hafez Avenue, Tehran 15914, Iran

http://www.aut.ac.ir/official/main.asp?uid=rajaieh 